\title{
La glucemia en ayunas como rastreo más simple para detectar la diabetes mellitus gestacional (DMG)
}

Using fasting plasma glucose concentrations to screen for gestational diabetes mellitus: prospective population based study. Perucchini D, Fisher U, Spinas G et al. BMJ. 1999 Sept 25; 319:812-15.

\section{Objetivo}

Comparar el dosaje de glucemia en ayunas con el test de carga con $50 \mathrm{~g}$ de glucosa (P50) para el diagnóstico de DMG, determinando el mejor punto de corte para ambos.

\section{Diseño}

Estudio prospectivo en población general.

\author{
Lugar \\ Hospital universitario de Zurich.
}

\section{Pacientes}

Desde 1995 a 1997 se reclutaron 520 embarazadas que cursaran embarazos simples (un solo feto), no presentaran antecedentes familiares de diabetes, fueran examinadas por un obstetra antes de la semana 24 de gestación y dieran su consentimiento informado. 328 (63\%) eran blancas, $99(19 \%)$ eran asiáticas, $31(6 \%)$ africanas y $62(12 \%)$ de otras razas. La edad promedio era de 28.4 (17-45) y su IMC promedio era de 23.8

\section{Descripción de los test diagnósticos}

Convencionalmente, el rastreo de la DMG en el control prenatal se realiza a través del dosaje de la glucemia una hora después de la administración de 50gr. de glucosa por vía oral (P50) en la semana 24-28 de gestación. Si esta es mayor o igual a $140 \mathrm{mg} / \mathrm{dl}$, se envía a la paciente a realizar un test diagnóstico que es la curva de tolerancia oral a la glucosa (CTOG).

En este estudio todas las pacientes realizaban la P50 entre la semana 24 y 28 de gestación, informando el tiempo desde la última ingesta. Todas ellas, realizaban a la semana una CTOG (test de referencia) sin importar el resultado de la P50. Se definió DMG si dos o más valores igualaban o superaban los valores de corte para la CTOG según Carpen-

\section{COMENTARIO}

En éste estudio, los autores proponen realizar rastreo de la DMG con una glucemia luego de 12 hs de ayuno en lugar de realizarlo con una P50 sólo en las pacientes con factores de riesgo (obesidad, edad materna a 30 años, historia familiar de DBT, raza, historia de malformaciones o macrosomias).

El estudio trata de responder una pregunta relevante: si rastrear con glucemia en ayunas es igual de eficaz que rastrear con la P50. Se utilizó un diseño prospectivo en la población general y un análisis a través de las curvas ROC* donde se comparan dos test (la glucemia en ayunas y la P50) con un test de referencia (la CTOG).

Sin embargo, el diseño del estudio no permite contestar esa pregunta. En primer lugar, no se describe de dónde saca el valor de la glucemia en ayunas. Es de suponer que se considera como glucemia en ayunas al primer valor de la CTOG. Como éste es el test de referencia y la glucemia en ayunas esta incluida dentro de los criterios diagnósticos de la DMG, es probable que esto pueda interferir con el análisis de los resultados (sesgo de medición).

El número de pacientes que presentaron DMG fue alto (10.2\% para una prevalencia gral. estimada del 3 al $5 \%$ ) y esto pudo haber sido influenciado por el porcentaje de mujeres asiáticas y africanas que ingresaron al estudio $(25 \%)$ que presentan una mayor incidencia de DMG. Un número tan elevado de éstas mujeres en la población de Suiza podría hacer pensar en un sesgo de selección. También llama la atención la baja sensibilidad ( $\mathrm{S}=59 \%$ ) que obtienen los autores para la P50 tomando como valor de cor*Ver glosario ter y Coustan del $4^{\circ}$ Workshop sobre DMG.

\section{Medición de resultados}

La relación entre las variables fue analizada con análisis univariado y se construyeron curvas ROC para analizar la sensibilidad y especificidad de los diferentes métodos. Sólo se incluyeron en el análisis a las mujeres que tuvieron su parto después de la semana 28 de gestación.

\section{Resultados}

Se diagnosticó DMG en 53 mujeres (10.2\%). Fue más frecuente en las mujeres asiáticas (16\%) y africanas (13\%) mientras que representó sólo el $8 \%$ en las mujeres blancas. La presentación de la DMG fue más común entre las mayores de 30 años ( $77 \%$ vs 46) y en las que presentaban mayor sobrepeso (IMC > 25: $54 \%$ vs $32 \%$ ).

Utilizando la curva ROC se determinó que el mejor valor de corte para rastrear DMG con una glucemia en ayunas era de $4.8 \mathrm{mmol} / \mathrm{L}(87 \mathrm{mg} / \mathrm{dl}$ ), observándose una sensibilidad (S) de $81 \%$ y una especificidad (E) de $76 \%$. Un 30\% de las embarazadas ( $n=155$ ) igualaban o sobrepasaban el punto de corte de $87 \mathrm{mg} / \mathrm{dl}$ y hubiesen tenido que realizar una CTOG. Cuando se analizó con la curva ROC a la P50, con el valor de corte de $7.8 \mathrm{mmol} / \mathrm{L}$ $(140 \mathrm{mg} / \mathrm{dl})$ se observó una $\mathrm{S}=59 \%$ y una $\mathrm{E}=91 \%$. El mejor valor de corte en esta curva fue de $7 \mathrm{mmol} / \mathrm{l}(126 \mathrm{mg} / \mathrm{dl})$, con una $\mathrm{S}=68 \%$ y una $\mathrm{E}=82 \%$. La prueba mejoraba su rendimiento si la mujer estaba con un ayuno mayor a 2 hs (la $S=100 \%$ y la $E=71 \%$ ).

\section{Conclusiones}

La glucemia en ayunas es un método simple y económico para rastrear la DMG. Se propone realizar el rastreo con una glucemia en ayunas entre la semana 24 y 28 de gestación. Si el valor de ésta es mayor a $4.8 \mathrm{mmol} / \mathrm{L}(87 \mathrm{mg} / \mathrm{dl})$ realizar directamente una CTOG. Esta estrategia evitaría que $70 \%$ de las pacientes realice una P50. te una glucemia a la hora de $140 \mathrm{mg} / \mathrm{dl}(7.8 \mathrm{mmol} / \mathrm{l})$ ya que en la mayoría de los estudios es del 71 al $83 \%$. Por último, la aplicabilidad del estudio en nuestro medio es discutible ya que no se define si las mujeres hispanas pertenecen al grupo de "otras" o se las incluye dentro del grupo de blancas. La principal controversia con el rastreo de la DMG no es cómo realizarlo sino definir si tiene sentido rastrear esta entidad. Hasta ahora ningún estudio bien realizado pudo demostrar que el tratamiento de la DMG disminuya la mortalidad perinatal. Aunque el tratamiento de la DMG con dieta e insulina reduce la incidencia de macrosomías, no disminuye el número de cesáreas ni de traumas en el parto. Actualmente tanto la US como la Canadian Task Force están de acuerdo en que el rastreo de la DMG carece de clara evidencia a favor o en contra (recomendación tipo C). El rastreo sólo esta recomendado por la American Diabetes Association a través de la prueba de desafío (P50), realizando a las que presentan una glucemia de más de $140 \mathrm{mg} \%$ a la hora, una CTOG.

Este estudio abre la posibilidad de un nuevo tipo de rastreo de la DMG, más simple y más económico que presenta como ventaja que no varía con la edad gestacional, presenta menos variaciones que las determinaciones post prandiales y es más extrapolable a diferentes poblaciones. De todas maneras, a pesar de existir un nuevo criterio diagnóstico de DBT fuera del embarazo, aún no se han determinado los criterios diagnósticos y de rastreo de la DMG.

\section{Referencias}

Coordinadora Servicio de Clínica Médica . Centro Medicus.

1. US Preventive Services Task Force. Guide to clinical preventive services: report of the U.S. Preventive Services. 2 ed. Baltimore: Williams \& Wilkins, c1996.

2. Zárate, M. Seguimiento del embarazo normal. En PROFAM: Programa de Educación a distancia de Medicina Familiar y Ambulatoria/ Esteban Rubinstein, dir. Buenos Aires: Hospital Italiano de Buenos Aires. Departamento de Docencia e Investigación, c1998. V.6, p 53-97.

3. Naylor C., Sermer M., Chen E., Farine D. Selective screening for gestational diabetes mellitus. N Engl J Med 1997; 337:1591-96.

4. Carpenter MW, Coustan DR. Criteria for screening tests fos gestational diabetes. Am J Obstet Gynecol 1982; 144: 768-73

5. Walkinshaw SA. Diet + insulin vs diet alone fos gestational diabetes. In : Enkin MW, KeirseMJNC, renfrew MJ, Neilson JP, eds. Pregnancy and childbirth module. Cochrane Database of systematic Reviews: Review N 06650, 20 april 1993, "Cochrane Updates on disk," 0xford: Update Software, 1994, Disk issue 1. 